\title{
Unbalance Compensation for AMB Systems with Input Delay: an Output Regulation Approach ${ }^{\text {光 }}$
}

\author{
Se Young Yoon ${ }^{\mathrm{a}, *}$, Long $\mathrm{Di}^{\mathrm{b}}$, Zongli Lin ${ }^{\mathrm{c}}$ \\ ${ }^{a}$ Department of Electrical and Computer Engineering, University of New Hampshire, Durham, NH 03824, USA \\ ${ }^{b}$ Department of Mechanical and Aerospace Engineering, University of Virginia, Charlottesville, VA 22904-4746, USA \\ ${ }^{c}$ Charles L. Brown Department of Electrical and Computer Engineering, University of Virginia, Charlottesville, VA 22904-4743, USA
}

\begin{abstract}
Unbalance compensation is an important technique for reducing rotor vibration in high speed rotating machines caused by residual rotor unbalance. As rotating machines in remote applications aim for higher speeds to gain efficiency and to reduce footprint, there is a need to extend the unbalance compensation techniques to active magnetic bearing (AMB) systems with delays in the control loop. This paper investigates the unbalance compensation problem for AMB systems with input delays. An unbalance compensation method is developed based on a solution to the output regulator problem for systems with input delay. The proposed unbalance compensation method is verified through simulation, and experimentally validated on an AMB test rig.
\end{abstract}

Keywords: time delay, output regulation, unbalance compensation, magnetic bearing, vibration control

\section{Introduction}

Active magnetic bearings (AMBs) are electromagnetic actuators for rotating machines. AMBs have seen steady growth in popularity over the past decades, starting from small turbomolecular pumps to larger compressors in the megawatt range. Using the magnetic forces generated by electric coils acting on the conductive material in the shaft, the AMBs levitate the rotor within the clearance of the bearing to allow machines to operate without any mechanical contact between the static and the rotating components. As a result, the rotating machine operates more efficiently and at higher speeds with negligible frictional losses, and for longer periods of time without servicing [1]. These capabilities are highly desirable for compressors, especially those in applications such as subsea oil and gas

\footnotetext{
This work was supported in part by the National Science Foundation under grants CMMI-1129752 and CMMI-1462171.

${ }^{*}$ Corresponding author

Email addresses: SeYoung.Yoon@unh.edu (Se Young Yoon), ld4vv@virginia.edu (Long Di), zl5y@virginia.edu (Zongli Lin)
}

Preprint submitted to Control Engineering Practice development, where the compressors are in harsh remote environments not easily accessible for frequent diagnosis and maintenance. Remote applications such as the subsea energy development have become technically and economically feasible with the availability of the AMB technology.

A technical challenge that needs to be addressed in the control of AMB systems in remotely operated compressors has to do with the communication delay introduced by the cabling system. Because the electronics driving the AMB actuators are sensitive to their environment, manufacturers many times choose to install the AMB control electronics at the control site, separated from the AMB actuators that are integrated to the remote machine. Long cables are then needed to connect the electronics to the actuators, which may add significant transmission delays in the control loop. Such delay may rapidly degrade the performance and stability of AMB systems, leading to undesired machine downtime, or even catastrophic machine failures. Therefore, the presence of input delays in the 
control of AMB systems needs to be explicitly addressed in the design of the rotor levitation controller.

The control of dynamic systems with time delay has been an active area of research. The predictor feedback control method is a popular approach among engineers and researchers for the control of systems with input delays. In this control method, a future state prediction is utilized in the computation of the control signal to cancel out the influence of the input delay. In other words, the delay system is transformed into an equivalent delay-free system by utilizing the state prediction in the control calculation. The majority of predictor feedback methods for linear systems are based on the Artstein model reduction technique [2] and the finite spectrum assignment technique [3]. Many variations of the predictor feedback control method have been developed in the literature over the years (see, for example, [4], [5] and [6]). A finite dimensional predictor, which is obtained by truncating the equation of traditional predictors, was developed in [7] for linear systems with all poles in the closed left-half plane. The truncated predictor relies on the low gain feedback design [8] to ensure the stability of the closed-loop system. This finite dimensional predictor was later expanded in [9] to systems with time-varying delays, and in [10] and [11] to exponentially unstable systems.

High speed rotating machines are also susceptible to large disturbance forces caused by rotor unbalances. A residual unbalance on a rotor can generate disturbance forces synchronous to the rotating speed, causing the rotor to go into a whirling motion. To reduce the effect that the rotor unbalance has on high-speed AMB systems, unbalance compensation, or autobalancing methods, have been studied and developed over the years. When designed and implemented properly, these methods can significantly reduce the disturbance forces acting on the rotor by allowing it to spin about its center of mass. The interest on rotor unbalance methods has increased rapidly in recent years as high speed AMB applications become more common, and the accessibility to digital controllers makes complex control algorithms easily implementable. A small sample of the literature studying the unbalance compensation problem can be found in [12-18] and the references therein. On the other hand, unbalance compensation methods have yet to be extended to AMB systems that are subjected to time delay.

This paper investigates an unbalance compensation problem for AMB systems with input delays. In particular, we derive and experimentally validate an unbalance compensation method based on the solution to an equivalent output regulation problem. Precise location and eccentricity of a rotor unbalance are difficult to measure in rotating machines. Instead, the locations of the unbalance forces in our mathematical model are strategically selected to reproduce the relevant rotor vibration patterns. The resulting model based unbalance compensation controller is demonstrated experimentally to significantly reduce the synchronous rotor vibrations and the magnitude of the AMB control input.

The remainder of this paper is structured as follows. The output regulation problem for systems with input delay is first introduced in Section 2, and solutions by both state-feedback and output-feedback are derived here. Next, the unbalance compensation problem for AMB systems is discussed in Section 3, and a control solution is proposed based on the output regulation method developed in Section 2. In Section 4 an AMB test rig with a flexible rotor is introduced. This test rig was constructed to reproduce the dynamics of AMB supported compressors commonly found in the oil and gas industry, and it will serve as the test platform for the unbalance compensation method proposed in this study. Simulation results for the disturbed AMB system with the proposed unbalance compensation method is presented in Section 5, and experimental results obtained from implementing the unbalance compensation method to the AMB test rig is presented in Section 6. Finally, concluding remarks are given in Sec- 
tion 7 .

\section{Output Regulation for Time Delay System}

Output regulation is one of the central problems in control theory. Its objective is to control the plant output such that it tracks a prescribed class of reference signals in the presence of disturbances. The reference signal to track in the output regulation problem, as well as the external disturbance input perturbing the system, are produced by an external generator known as the exosystem. The output regulation problem has been studied extensively since it was formulated by [19] for linear systems, and by [20] for nonlinear systems. A comprehensive treatment of the nonlinear output regulation problem can be found in [21], [22] and [23].

A few works studying the output regulation problem for time delay systems can be found in the literature. The output regulation problem for systems with state delays was discussed in [24] and [25], where the authors derive the necessary and sufficient conditions for the existence of a solution by employing a similar argument as presented in [19] for the delay-free case. In the nonlinear setting, [26] extends the conditions for solvability of the output regulation problem to systems with state delays. For systems with input delay, [27] introduces a robust solution to the output regulation problem. For linear time-invariant and continuous systems with delays in the states, inputs and outputs, the output regulation problem was studied in $[28,29]$.

\subsection{Definition of the Output Regulation Problem}

Consider a linear time-invariant system with input delay,

$$
\begin{aligned}
& \dot{x}(t)=A x(t)+B u(t-\tau)+\mathcal{P} w(t), \\
& y(t)=C x(t),
\end{aligned}
$$

with state vector $x \in \mathbf{R}^{n}$, input vector $u \in \mathbf{R}^{m}$, output vector $y \in \mathbf{R}^{r}$, and input delay $\tau \geq 0$. The external disturbance $w \in \mathbf{R}^{2 s}$ is generated by an exosystem

$$
\dot{w}(t)=\mathcal{S} w(t)
$$

In general, $w$ could be of any dimension. However, its dimension has been restricted to an even number since the disturbances from the rotor unbalance forces to be considered are sinusoidal. In the output regulation problem studied in this paper, we wish to find a control law that regulates the error signal defined as

$$
e(t)=\mathcal{C} x(t)+\mathcal{D} u(t-\tau)+\mathcal{Q} w(t) .
$$

Then, the objective of our control problem is to find a control law such that

1. the closed-loop system is asymptotically stable when $w \equiv 0$, and

2. for any arbitrary initial conditions of $x$ and $w$, the error $e(t)$ approaches zero as $t \rightarrow \infty$.

Some standard assumptions are made on the system (1) that are required for the solvability of the output regulation problem.

Assumption 1. The eigenvalues of $\mathcal{S}$ have nonnegative real parts.

Assumption 2. The system (1) with $w \equiv 0$ is detectable and stabilizable.

Assumption 1 does not affect the generality of the problem since asymptotically stable eigenvalues of $\mathcal{S}$ do not affect the regulation of the output. Assumption 2 is required for the existence of a control law that asymptotically stabilizes system (1), when $w \equiv 0$.

\subsection{Output Regulation by State Feedback}

The following lemma provides a solution to the output regulator problem by state feedback, given that a stabilizing control law exists for the disturbance-free system. 
Lemma 1. Consider the time-delay system (1) satisfying Assumptions 1 and 2, and let the state feedback law $u(t)=\kappa x(t), \kappa \in \mathbf{R}^{m \times n}$, be an asymptotically stabilizing control law for (1) when $w \equiv 0$. Then, the output regulation control

$$
u(t)=\nu(t)+\Gamma e^{\tau \mathcal{S}} w(t)
$$

where $\nu(t)=\kappa z(t)$ and $z(t)=x(t)-\Pi w(t)$, satisfies the objectives of the output regulation problem if and only if there exist matrices $\Pi \in \mathbf{R}^{n \times 2 s}$ and $\Gamma \in \mathbf{R}^{m \times 2 s}$ that satisfy the regulator equations

$$
\begin{aligned}
\Pi \mathcal{S} & =A \Pi+B \Gamma+\mathcal{P}, \\
0 & =\mathcal{C} \Pi+\mathcal{D} \Gamma+\mathcal{Q} .
\end{aligned}
$$

Proof. The above is a special case of a similar lemma in [29]. The proof comes readily from defining the state transformation $z(t)=x(t)-\Pi w(t)$, and the input to be in the form of (4). Let $\Pi$ and $\Gamma$ be the solution to (5). Then (1) simplifies to

$$
\begin{aligned}
& \dot{z}(t)=A z(t)+B \nu(t-\tau), \\
& e(t)=\mathcal{C} z(t)+\mathcal{D} \nu(t-\tau),
\end{aligned}
$$

which is equivalent to (1) with $w \equiv 0$. Assumption 2 yields that there exists a stabilizing state-feedback control law $\nu(t)=\kappa z(t)$, and the fact that $\lim _{t \rightarrow \infty} e(t)=0$ leads to the conclusion that (4) is a solution to the output regulation problem.

To demonstrate the necessary condition, assume that (4) is a solution to the output regulation problem. Then, the tracking error in (3) becomes

$$
e(t)=\mathcal{C} z(t)+\mathcal{D} \kappa z(t-\tau)+(\mathcal{C} \Pi+\mathcal{D} \Gamma+\mathcal{Q}) w(t)
$$

which must satisfy that $\lim _{t \rightarrow \infty} e(t)=0$. Since Assumption 1 states that $w(t)$ is nonvanishing, the tracking error approaches zero for arbitrary initial conditions if $\lim _{t \rightarrow \infty} z(t)=$ 0 and $(5 \mathrm{~b})$ is true. Furthermore, it was assumed that $\nu(t)=\kappa z(t)$ asymptotically stabilizes (6). For $\lim _{t \rightarrow \infty} z(t)=$ 0 to be true under Assumption 1, it is required that (5a) is satisfied.
A stabilizing truncated predictor feedback (TPF) control law for linear systems with input delay was derived in [30] to maximize the tolerable delay bound. In particular, this control law was experimentally validated on a test rig that was built to closely represent AMB systems in industrial machines. For the undisturbed system (1) with $w \equiv 0$, the TPF control takes the form of a finite dimensional predictor feedback law as discussed in [7] and $[9]$

$$
u(t)=K \mathrm{e}^{A \tau} x(t)
$$

where $K$ is a feedback gain to be found.

Lemma 2 ([11]). Consider the undisturbed input delayed system (1) with $w \equiv 0$, where the pair $(A, B)$ is controllable. Given some positive definite matrix $W$, the TPF control (7) with

$$
K=-B^{T} W^{-1}
$$

asymptotically stabilizes the input delayed system if there exist real scalars $\beta>0$ and $\alpha>0$ such that

$$
\begin{aligned}
& \alpha W \geq B B^{T}, \\
&\left(A-\frac{1}{2} \beta I\right) W+W\left(A-\frac{1}{2} \beta I\right)^{T}<0, \\
& W\left(A+\frac{\alpha^{3} \bar{D}}{2 \beta} e^{\beta \bar{D}}\left(e^{\beta \bar{D}}-1\right) I\right)^{T} \\
&+\left(A+\frac{\alpha^{3} \bar{D}}{2 \beta} e^{\beta \bar{D}}\left(e^{\beta \bar{D}}-1\right) I\right) W<B B^{T} .
\end{aligned}
$$

The proof of Lemma 2 and a numerical method for solving the nonlinear matrix inequality conditions (9) can be found in [11] and [30]. Finally, the combined results of Lemmas 1 and 2 provide us with a solution to the state feedback output regulation problem.

\subsection{Output Regulation by Output Feedback}

Consider the combined perturbed system with $\chi=$ $\left[x^{\mathrm{T}} w^{\mathrm{T}}\right]^{\mathrm{T}}$, and

$$
\begin{aligned}
\dot{\chi}(t) & =\left[\begin{array}{ll}
A & \mathcal{P} \\
0 & \mathcal{S}
\end{array}\right] \chi(t)+\left[\begin{array}{l}
B \\
0
\end{array}\right] u(t-\tau), \\
y(t) & =\left[\begin{array}{ll}
C & 0
\end{array}\right] \chi(t), \\
e(t) & =\left[\begin{array}{ll}
\mathcal{C} & \mathcal{Q}
\end{array}\right] \chi(t)+D u(t-\tau) .
\end{aligned}
$$


Lemma 3. Consider the time-delay system (10) satisfying Assumptions 1 and 2, and let the state feedback law

$$
u(t)=\kappa z(t)+\Gamma e^{\tau \mathcal{S}} w(t)
$$

as defined in Lemma 1, be a solution to the problem of output regulation by state feedback, where $z(t)=x(t)-\Pi w(t)$, and $\Pi \in \mathbf{R}^{n \times 2 s}$ and $\Gamma \in \mathbf{R}^{m \times 2 s}$ satisfy the regulator equations in (5). Then, the output feedback control law

$$
\begin{aligned}
& \dot{\hat{\chi}}(t)=\mathbb{A} \hat{\chi}(t)+\mathbb{B} u(t-\tau)+L(\mathbb{C} \hat{\chi}(t)-y(t)),
\end{aligned}
$$

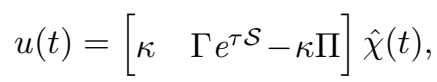

satisfies the objectives of the output regulation problem, where

$$
\hat{\chi}=\left[\begin{array}{l}
\hat{x} \\
\hat{w}
\end{array}\right], \mathbb{A}=\left[\begin{array}{ll}
A & \mathcal{P} \\
0 & \mathcal{S}
\end{array}\right], \mathbb{B}=\left[\begin{array}{l}
B \\
0
\end{array}\right], \mathbb{C}=\left[\begin{array}{ll}
C & 0
\end{array}\right],
$$

if there is a matrix $L \in \mathbf{R}^{r \times m}$ such that $(\mathbb{A}+L \mathbb{C})$ is Hurwitz.

Proof. Similarly to the proof of Lemma 1, we define the state transformation $z(t)=x(t)-\Pi w(t)$, and let $\hat{z}=$ $\hat{x}-\Pi \hat{w}$ and $\tilde{z}=\hat{z}-z$. The closed-loop equation of the $z$ system becomes

$$
\begin{array}{rl}
\dot{z}(t)=A & z(t)+B \kappa z(t-\tau) \\
+ & {\left[\begin{array}{ll}
B \kappa & B\left(\Gamma \mathrm{e}^{\tau \mathcal{S}}-\kappa \Pi\right)
\end{array}\right] \tilde{\chi}(t-\tau),} \\
e(t)=\mathcal{C} z(t)+\mathcal{D} \kappa z(t-\tau) \\
+\left[\begin{array}{ll}
\mathcal{D} \kappa & \mathcal{D}\left(\Gamma \mathrm{e}^{\tau \mathcal{S}}-\kappa \Pi\right)
\end{array}\right] \tilde{\chi}(t-\tau),
\end{array}
$$

where the equation for $\tilde{\chi}=\hat{\chi}-\chi$ is obtained from (10) and (11) as

$$
\dot{\tilde{\chi}}(t)=(\mathbb{A}+L \mathbb{C}) \tilde{\chi}(t)
$$

We note that the $z(t)$ and $\tilde{\chi}(t)$ subsystems are decoupled, and (13) is asymptotically stable. Lemma 1 also yields that $z(t)$ asymptotically approaches zero when $\tilde{\chi} \equiv 0$. Finally, as the states $z(t)$ and $\tilde{\chi}(t)$ asymptotically approach zero, the limit of the tracking error signal will also converge to zero, or $\lim _{t \rightarrow \infty} e(t)=0$. Therefore, (11) is a solution to the output regulation problem.

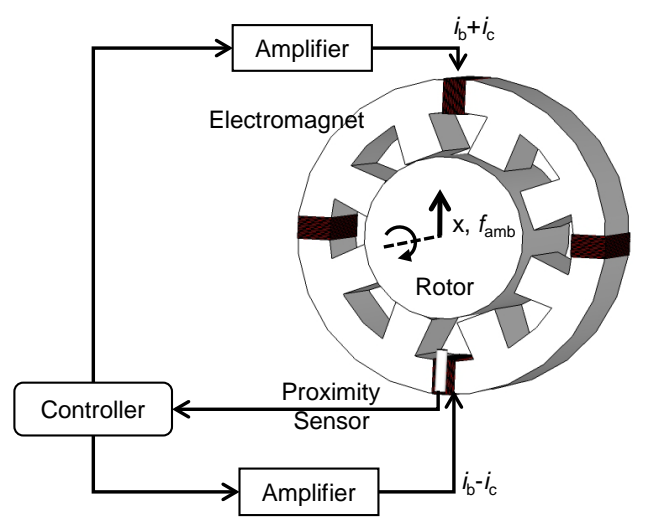

Figure 1: AMB operating principle.

\section{Unbalance Compensation in AMB Systems}

\subsection{Active Magnetic Bearings}

Active magnetic bearings (AMBs) are electromagnetic actuators that provide non-contact support to the rotor. Compressors with AMBs benefit from high efficiency, reliability and energy density, as well as having the capability to actively stabilize the internal flow [31]. Built in a ring configuration, AMB actuators use electromagnetic forces to levitate the rotor within the bearing clearance. The basic AMB operating principle in one control axis is illustrated in Fig. 1. An actuator of the AMB system is a pair of electromagnets acting on opposite sides of the target rotor. Each electromagnet operates on a fixed bias current $i_{\mathrm{b}}$, perturbed by a control current $i_{\mathrm{c}}$. The net force generated by the pair of AMB actuators can be approximated by

$$
f_{\mathrm{amb}}=K_{\mathrm{x}} x+K_{\mathrm{i}} i_{\mathrm{c}},
$$

where $K_{\mathrm{x}}$ is the open loop stiffness and $K_{\mathrm{i}}$ is the open loop gain of the AMB.

A rotor levitation controller calculates a control command to the actuators based on the feedback signal from the sensors measuring the rotor displacement. The command signal from the levitation controller is forwarded to the current amplifiers powering the coils of the AMB actuators, thus closing the control loop. The control objective of the closed-loop AMB system is to keep the rotor lev- 
itated near the bearing geometric center. The combined system of AMB actuators, electronics, target rotor, and controller is commonly referred as the AMB system.

\subsection{AMB System Modeling}

The lateral dynamic equation of the AMB system, under the assumption that the rotor and AMB actuators are symmetric about both the $x$ and $y$ lateral axes, can be expressed in the state space form as

$$
\begin{aligned}
{\left[\begin{array}{c}
\dot{\zeta}_{x}(t) \\
\dot{\zeta}_{y}(t)
\end{array}\right]=} & {\left[\begin{array}{cc}
A_{\zeta} & \omega G \\
-\omega G & A_{\zeta}
\end{array}\right]\left[\begin{array}{c}
\zeta_{x}(t) \\
\zeta_{y}(t)
\end{array}\right]+\left[\begin{array}{cc}
B_{\mathrm{c}} & 0 \\
0 & B_{\mathrm{c}}
\end{array}\right]\left[\begin{array}{l}
i_{\mathrm{c}, x}(t-\tau) \\
i_{\mathrm{c}, y}(t-\tau)
\end{array}\right] } \\
& +\sum_{j=1}^{s}\left[\begin{array}{cc}
B_{\mathrm{d}, j} & 0 \\
0 & B_{\mathrm{d}, j}
\end{array}\right]\left[\begin{array}{l}
w_{x, j}(t) \\
w_{y, j}(t)
\end{array}\right]
\end{aligned}
$$

where the subscripts $x$ and $y$ refer to the lateral $x$ and $y$ directions of the AMB system, respectively, $i_{\mathrm{c}}$ is the control current to the AMBs, and $\omega$ represents the rotating speed. The state vector $\zeta$ describes the lateral dynamics of the AMB system, which includes the AMB actuators, AMB electronics and rotor. The lateral dynamics of the rotor are commonly modeled through finite-element methods, and modal coordinate transformation is employed to simplify the resulting differential equation. The matrices $A_{\zeta} \in \mathbf{R}^{20 \times 20}$ and $B_{\mathrm{c}} \in \mathbf{R}^{20 \times 2}$ are the state space matrices of a single axis AMB system, and the lateral axes are coupled by the gyroscopic effect $\omega G$. The disturbance forces generated by the $j^{\text {th }}$ unbalance mass on the rotor are represented by $w_{x, j}$ and $w_{y, j}$, and they are found to be of the form

$$
\begin{aligned}
& w_{x, j}=\epsilon_{j} \omega^{2} \cos \left(\omega t+\theta_{j}\right), \\
& w_{y, j}=\epsilon_{j} \omega^{2} \sin \left(\omega t+\theta_{j}\right),
\end{aligned}
$$

where $\epsilon_{j}$ is the unbalance eccentricity and $\theta_{j}$ is the phase angle. The above sinusoidal disturbances can be modeled by a dynamic system,

$$
\left[\begin{array}{c}
\dot{w}_{x, j} \\
\dot{w}_{y, j}
\end{array}\right]=\left[\begin{array}{cc}
0 & -\omega \\
\omega & 0
\end{array}\right]\left[\begin{array}{l}
w_{x, j} \\
w_{y, j}
\end{array}\right] .
$$

After grouping the states $\zeta=\left[\zeta_{x} \zeta_{y}\right]^{\mathrm{T}}$, control inputs $i_{\mathrm{c}}=$ $\left[\begin{array}{ll}i_{\mathrm{c}, x} & i_{\mathrm{c}, y}\end{array}\right]^{\mathrm{T}}$ and disturbance forces $w_{j}=\left[\begin{array}{ll}w_{x, j} & w_{y, j}\end{array}\right]^{\mathrm{T}}$, the state space equations in (15) and (17) can be rewritten in the form

$$
\begin{aligned}
\dot{\zeta}(t) & =\mathbf{A} \zeta(t)+\mathbf{B} i_{\mathrm{c}}(t-\tau)+\sum_{j=1}^{s} \mathbf{P}_{j} w_{j}(t), \\
\dot{w}_{j}(t) & =\mathbf{S} w_{j}, \text { for } j=1,2, \cdots, s .
\end{aligned}
$$

Finally, let $\omega^{\mathrm{T}}=\left[\omega_{1}^{\mathrm{T}} \omega_{2}^{\mathrm{T}} \cdots \omega_{s}^{\mathrm{T}}\right]$ to convert the above system to the form given in (1) and (2).

\subsection{Unbalance Compensation}

In developing our unbalance compensation control, it is assumed that the rotor is a rigid body. In other words, we focus on the vibration patterns composed of the rigid body modes of the rotor as shown in Fig. 2. The parallel mode is characterized by the translation of the rotor about the center line. On the other hand, the conical mode describes the rotor tilt about its center of mass. In a sub-critical AMB system operating below its first flexible mode frequency, the rotor vibration profile will follow a combination of the two rigid body modes described above.

In order to simultaneously regulate both parallel and conical modes of the rotor, two locations along the rotor length are selected where we will assume the disturbances $\omega_{j}$ 's are applied. These locations are selected such that they do not coincide with the nodal points of the rotor modes under consideration. In the case where higher order modes of the rotor are considered, additional disturbance input locations may be introduced. For simplicity, the locations of the disturbance forces are defined to match the locations of the AMB actuators. Based on this information, the matrices $\mathbf{P}_{1}$ and $\mathbf{P}_{2}$ can be found during the modeling of the rotor dynamics.

In this study we consider two different cases of the output regulation problem, corresponding to two separate error signals defined for the AMB system. In the first case, the error signal is the rotor displacement $d=\left[\begin{array}{ll}d_{x} & d_{y}\end{array}\right]^{\mathrm{T}}$, 


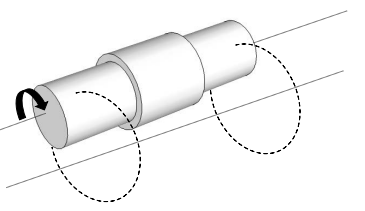

(a) Parallel mode

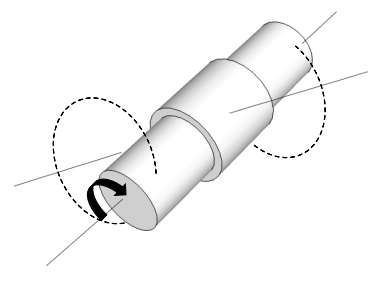

(b) Conical mode
Figure 2: Rotor rigid body modes.

measured at the AMB sensor locations. The displacement can be defined from the AMB system states as

$$
d=\mathbf{C}_{\text {sens }} \zeta
$$

where the output matrix $\mathbf{C}_{\text {sens }}$ is obtained during the derivation of the state space equation of the AMB systems. By regulating the rotor displacement, the observed rotor vibration is reduced, and the rotor is forced to rotate about its geometric center.

In the second case of the output regulation problem, the error signal is defined to be the applied AMB forces,

$$
f_{\mathrm{amb}}=K_{\mathrm{x}} \mathbf{C}_{\mathrm{amb}} \zeta+K_{\mathrm{i}} i_{\mathrm{c}}
$$

Once again, the matrix $\mathbf{C}_{\mathrm{amb}}$ is obtained such that $\mathbf{C}_{\mathrm{amb}} \boldsymbol{\zeta}$ corresponds to the rotor displacements at the bearing locations. In this case, the AMB forces are regulated, which in turn reduce the disturbance forces generated by the unbalance mass on the rotor [32]. Based on (16), the unbalance force is zero only if the unbalance eccentricity equals zero,

$$
\epsilon_{j}=0, \text { for } j=1,2, \cdots, s,
$$

and the rotor rotates about its center of mass. Therefore, this second case corresponds to a compensation of the rotor unbalance.

\section{Experimental Setup}

This section introduces the flexible rotor AMB test rig [33], as shown in Fig. 3. This test rig was constructed to accurately reproduce the AMB system dynamics in an industrial high speed centrifugal gas compressor and has

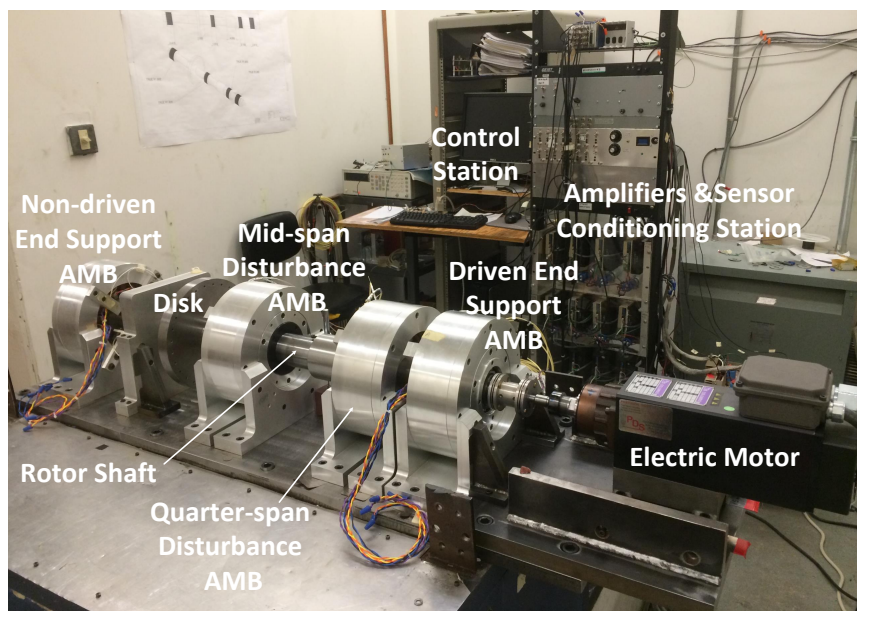

Figure 3: AMB test rig with flexible rotor.

been used to carry out studies on the AMB systems. Some of the most difficult challenges in the control of AMB systems are captured by the test rig, including flexible rotor modes, unbalance forces, gyroscopic moments and crosscoupling stiffness. Furthermore, in order to reproduce the latency in the control signal in remote turbomachinery applications, a time delay can be added to the output of the AMB rotor levitation controller.

\subsection{Active Magnetic Bearings}

The AMB test rig shown in Fig. 3 has two radial AMBs, with actuators located respectively at the driven end (DE) and the nondriven end (NDE) of the rotor. Each AMB actuator was built in a 16-pole heteropolar configuration and has a nominal air gap of $0.381 \mathrm{~mm}$ (15 mils) and a saturation flux density of $1.2 \mathrm{~T}$. For the linearization of the AMB forces, the bias current was selected to be $3.8 \mathrm{~A}$ for all actuators, which corresponds to a static load capacity of $1336 \mathrm{~N}$ per control axis. Two backup rolling element bearings are also mounted together with the AMBs. They are designed to contain the rotor displacement in the event of an AMB failure. They prevent an accidental contact between the rotor and the bearing stator, and hence potential damages to the AMB system. The nominal radial clearance of these backup bearings is $0.254 \mathrm{~mm}$ (10 mils). 


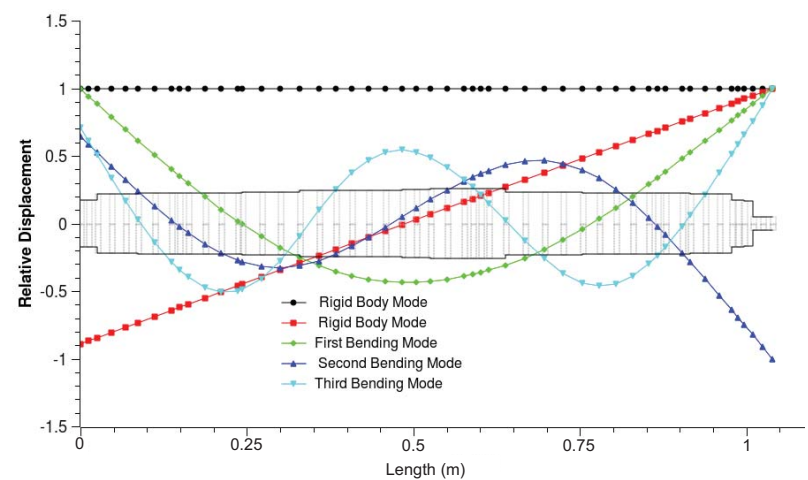

Figure 4: The free-free mode shape plot of the rotor.

\subsection{Rotor}

The target of the AMB actuator is the rotor. The AMB test rig as shown in Fig. 3 has a rotor constructed of carbon steel. The rotor is $1.23 \mathrm{~m}$ in length, with an average shaft radius of around $30 \mathrm{~mm}$ and total weight of $44.9 \mathrm{~kg}$. The rotor length, diameter and other design paramenters were selected to be similar to those of rotors in industrial gas compressors.

Rotors in high-speed machines, such as in this AMB test rig, have resonance modes with frequencies within their operating speed range. These modes may be excited during the normal operation of the machine as the rotor speed dwells near the resonance frequencies. The mode shapes corresponding to the first five modes of the rotor in the AMB test rig are presented in Fig. 4. The mode shapes give essential information of the rotor vibration pattern when different modes are excited.

\subsection{Controller Hardware}

AMB systems are open-loop unstable, and feedback control is required for rotor levitation. The feedback measurement for control is the rotor displacement, which is monitored by Kaman eddy current sensors located adjacent to the AMB actuators. For each AMB control axis, there are one pair of sensor probes arranged in a differential setup. The control signal calculated by the levitation controller is forwarded to current amplifiers powering the

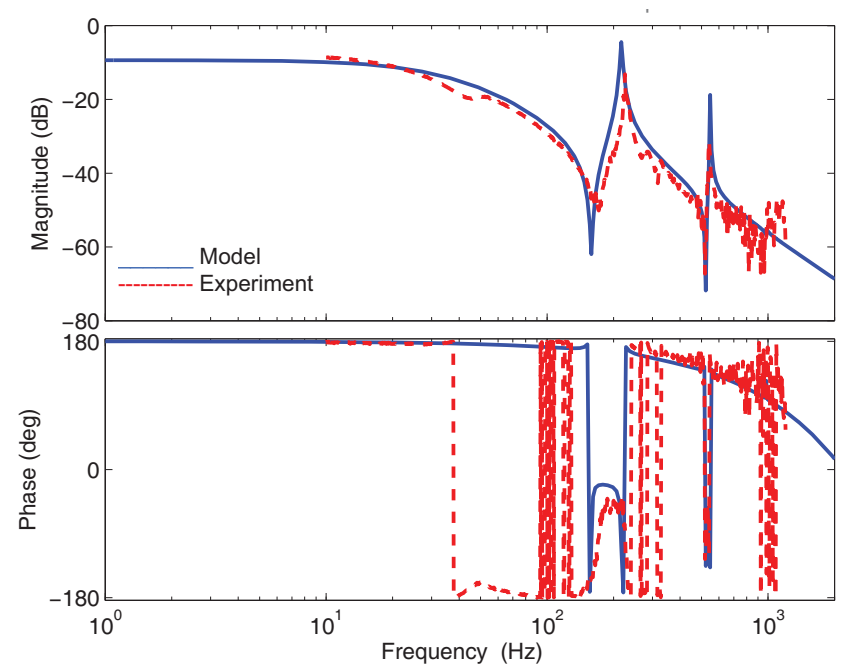

Figure 5: Bode plots for the test rig: analytic model vs experimental measurements.

AMB actuators. There are four Model 422 PWM amplifiers from Copley Controls for each side of the AMB actuators, and each amplifier generates a continuous current output up to $10 \mathrm{~A}$.

The rotor levitation controller for the AMB system is implemented on an Innovative Integration M6713 digital signal processing (DSP) board at a sampling frequency of $12 \mathrm{kHz}$. The DPS board has a $300 \mathrm{Mhz}$ 32-bit floating point processor with 16 analog input-output channels. The control algorithm is implemented in $\mathrm{C}++$.

\subsection{AMB System Unbalance Compensation Controller}

The mathematical model of the assembled AMB system is validated with measurements obtained from the AMB test rig. Figure 5 compares the Bode plots of the AMB system obtained from the theoretical model and the measured response. The Bode plots show close agreement between theory and experiment, and validates our mathematical model of the AMB system.

Based on the validated AMB system model, the rotor levitation controller and the unbalance compensation methods investigated in this paper were designed. The rotor levitation controller was designed for the undisturbed system $(\omega \equiv 0)$ following the results of Lemma 2 . A 


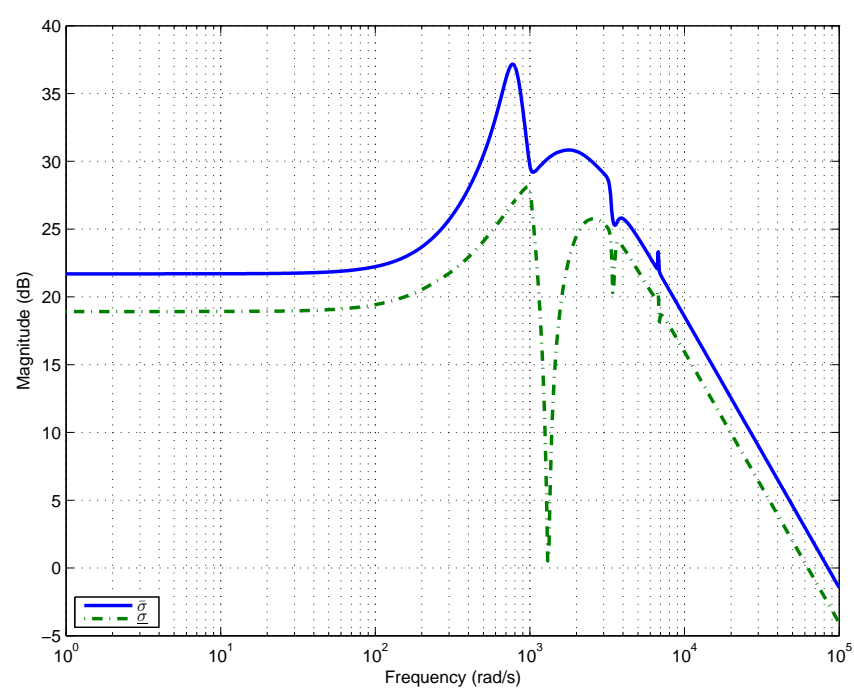

Figure 6: Maximum and minimum singular value plot of the rotor levitation controller for the undisturbed system.

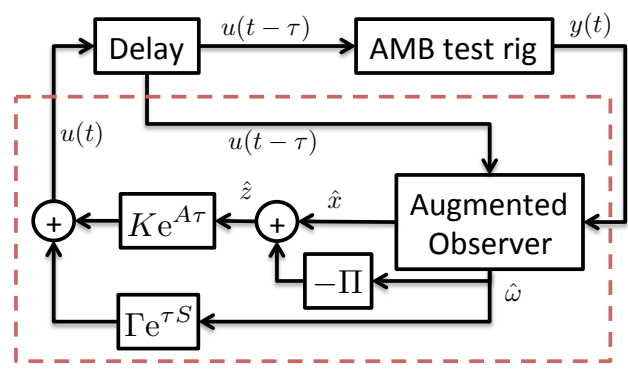

Figure 7: Block diagram of the controller implementation.

levitation controller satisfying the stability conditions in Lemma 2 was previously studied in [30], and experiments demonstrated that the control law can stabilize the undisturbed AMB system for delays up to ten times the sampling time of the digital controller $(0.83 \mathrm{~ms})$. The maximum and minimum singular values of the resulting output feedback control law is shown in Fig. 6. For the unbalance compensation control, the results of Lemma 3 yielded an augmented observer to estimate both the plant states and the exosystem states corresponding to the unbalance forces. The augmented observer was utilized to construct the output feedback control solution in (11). The implementation of the final controller is illustrated in Fig. 7.

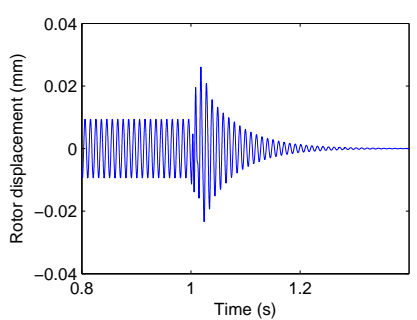

(a) Non-driven end

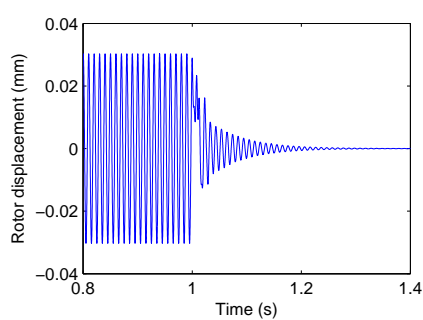

(b) Driven end
Figure 8: Simulated rotor displacements at the AMB locations during the regulation of the measured rotor displacement.

\section{Simulation Results}

The output regulation control laws designed for the AMB test rig with the regulated error signals (19) and (20) were tested through simulation. The simulation of the AMB system included two rotor unbalance masses located at the locations of the bearings. The rotating speed in the simulation was fixed at $6,000 \mathrm{rpm}$, and the input delay was set to $\tau=0.5 \mathrm{~ms}$.

First, the rotor displacement regulation control was tested, and the simulation results for a lateral axis are presented in Fig. 8. The AMB system is initially controlled by the levitation controller designed for the undisturbed system, and the output regulator component is only activated near time $t=1 \mathrm{~s}$. As expected, the amplitude of the rotor vibration shown in Fig. 8 approaches zero after the output regulation control is activated.

Next, the AMB force regulation controller was tested, and the resulting simulation responses are presented in Fig. 9. As in the previous case, the output regulation controller is engaged near time $t=1 \mathrm{~s}$. The amplitude of the AMB forces shown in Fig. 9 converges to zero after the unbalance compensation method is activated. This corresponds to a balanced rotor rotating about its center of mass and with zero unbalance force disturbances.

\section{Experimental Testing}

The output regulation control laws verified in the previous section were implemented and tested on the $\mathrm{AMB}$ 


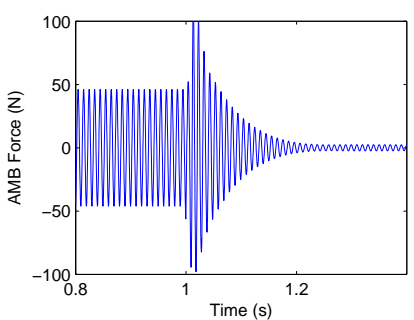

(a) Non-driven end

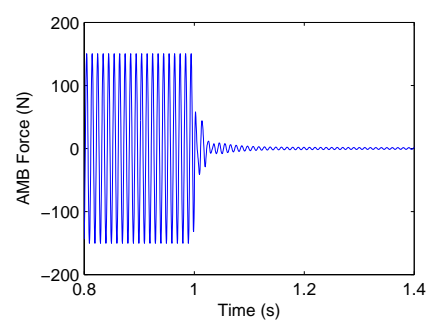

(b) Driven end

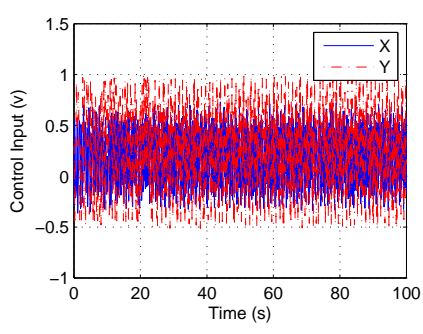

(a) Non-driven end

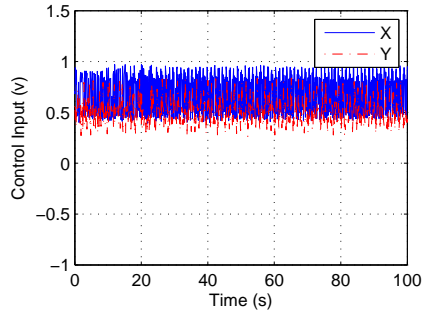

(b) Driven end
Figure 9: Simulated AMB forces during the regulation of the AMB force.

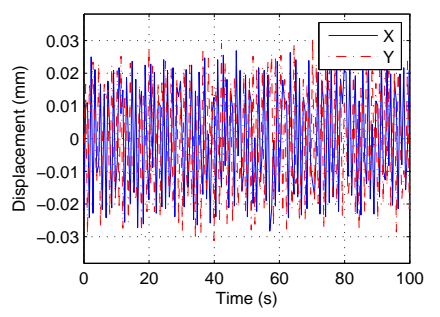

(a) Non-driven end

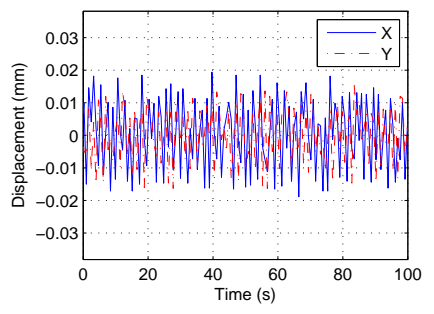

(b) Driven end
Figure 10: Measured rotor displacements at the AMB locations with the rotor levitation controller designed for the undisturbed system.

test rig described in Sect. 4. As in the simulation, the rotating speed of the $\mathrm{AMB}$ system is $6,000 \mathrm{rpm}$, and the input delay is $\tau=0.5 \mathrm{~ms}$. The eccentricity and axial distribution of the rotor unbalances are unknown, and they are product of residual errors during the rotor balancing process.

Figures 10, 11 and 12 present the results corresponding to the AMB system under the rotor levitation control law designed for the undisturbed system $(\omega \equiv 0)$, which will serve as the base case to compare the performance of the output regulation controllers. The rotor vibrations measured by the sensors at the bearing locations are presented in Fig. 10. The control signals from the controller to the current amplifiers powering the AMB actuators are presented in Fig. 11. Lastly, the orbits of the rotor displacements at the bearing locations are illustrated in Fig. 12.

Next, we present the results corresponding to the AMB system under the rotor position regulation control law in Figs. 13, 14 and 15. The error signal to regulate is the rotor displacement measurements by sensors at the AMB
Figure 11: Measured AMB control voltages with the rotor levitation controller designed for the undisturbed system.

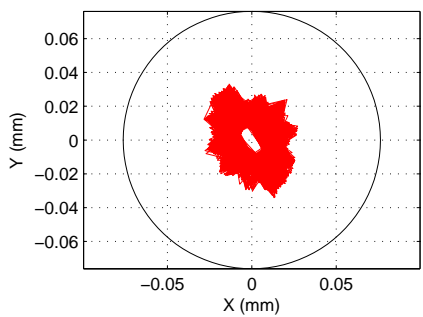

(a) Non-driven end

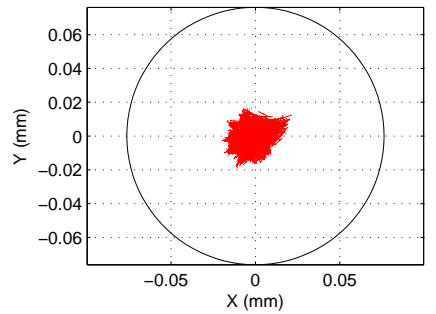

(b) Driven end
Figure 12: Measured rotor orbits at the AMB locations with the rotor levitation controller designed for the undisturbed system.

location. Fig. 13 shows the rotor vibrations measurement near the location of the bearings. It is clear that the rotor vibration level has been significantly reduced when compared to Fig. 10. The control voltages to the current amplifiers in Fig. 14 also show smaller amplitude oscillations compared to the base case, but the improvement is not as significant. The rotor orbits at the bearing locations with the rotor displacement regulation controller are presented in Fig. 15.

Finally, Figs. 16, 17 and 18 show the measured response

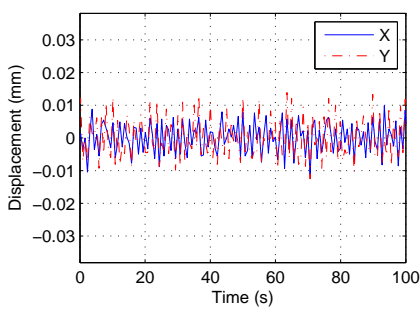

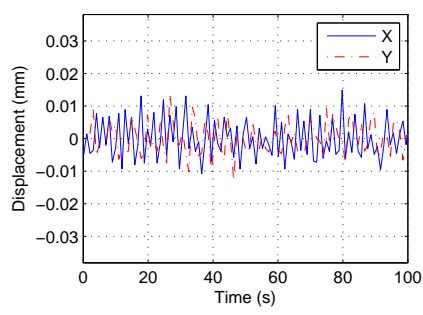

(b) Driven end
Figure 13: Measured rotor displacements at the AMB locations during the regulation of the rotor displacement. (a) Non-driven end 


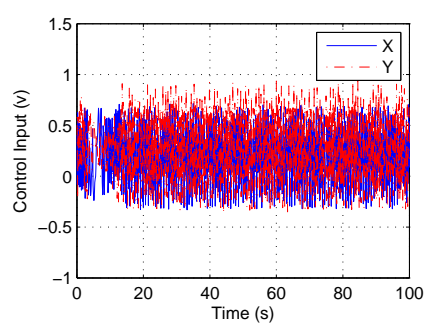

(a) Non-driven end

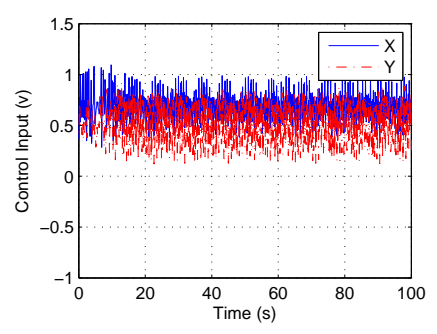

(b) Driven end
Figure 14: Measured AMB control voltages during the regulation of the rotor displacement.

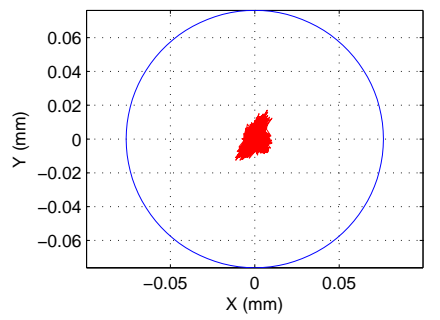

(a) Non-driven end

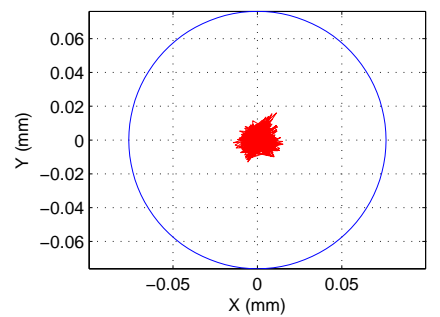

(b) Driven end
Figure 15: Measured rotor orbits at the AMB locations during the regulation of the rotor displacement.

of the AMB system under the unbalance compensation control law, where the objective is to regulate the AMB force estimates. The measured rotor displacements at the AMB sensor locations in Fig. 16 demonstrate that the rotor vibration level is well contained, comparable to the results in Fig. 13. The most significant improvement can be seen in the control voltages to the AMB current amplifiers in Fig. 17, which shows that the input energy required to compensate for the unbalance forces and keep the rotor levitated was significantly reduced. The unbalance forces disturbing the AMB system decrease as the mass center of the rotor approaches the axis of rotation. Finally, the rotor position orbits at the bearing locations are shown in Fig. 18.

\section{Conclusions}

This paper investigated the unbalance compensation problem for AMB systems in the presence of input delay. First the output regulation problem was presented for sys-

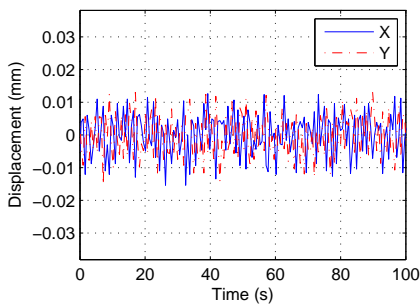

(a) Non-driven end

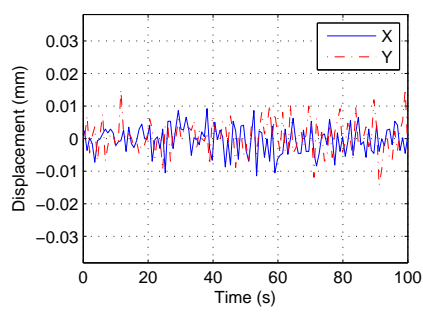

(b) Driven end
Figure 16: Measured rotor displacements at the AMB locations during the regulation of the AMB forces.

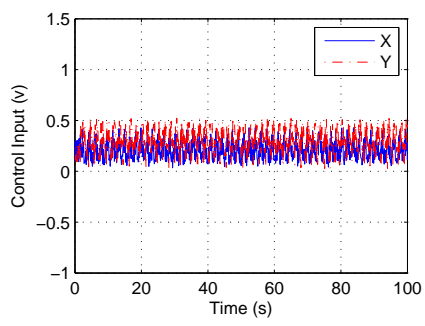

(a) Non-driven end

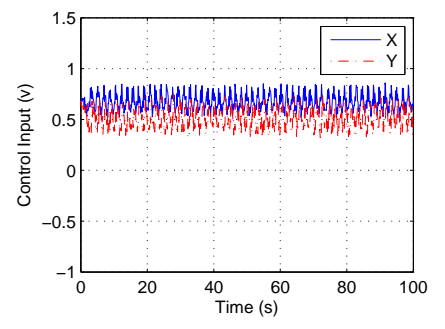

(b) Driven end
Figure 17: Measured AMB control voltages during the regulation of the AMB forces.

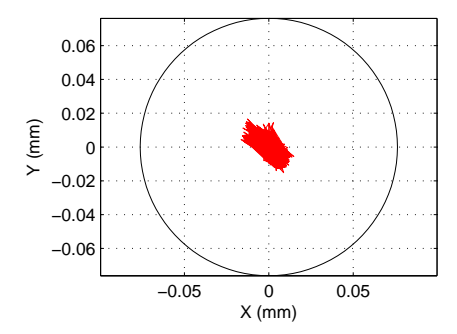

(a) Non-driven end

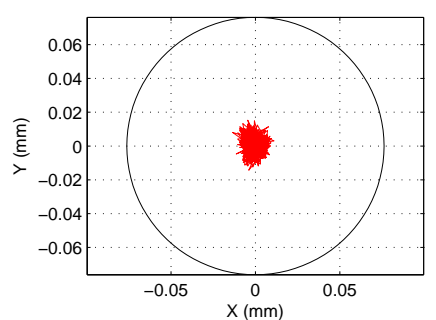

(b) Driven end
Figure 18: Measured rotor orbits at the AMB locations during the regulation of the AMB forces. 
tems with input delay, and an output feedback solution was constructed from a truncated predictor feedback stabilizing control. Next, an unbalance compensation method was developed based on our solution to the output regulation problem. Using an AMB test rig, which was constructed to capture all the relevant rotordynamic characteristics found in industrial gas compressors, two unbalance compensation controllers were designed and tested in both simulation and experiment. The first unbalance compensation controller was designed to reduce the rotor vibration by regulating the rotor displacement, while the second unbalance compensation controller was designed to reduce the unbalance forces acting on the rotor by regulating the AMB control forces. Both simulation and experimental results demonstrate that the proposed unbalance compensation methods are effective in suppressing the rotor vibrations and reducing the peak control input.

\section{References}

[1] G. Schweitzer, E. Maslen (Eds.), Magnetic Bearings, Springer, 2009.

[2] Z. Artstein, Linear systems with delayed controls: a reduction, IEEE Trans. Autom. Control 27 (1982) 869-876.

[3] A. Z. Manitius, A. W. Olbrot, Finite spectrum assignment problem for systems with delays, IEEE Trans. Autom. Control 24 (1979) 541-553.

[4] N. Sharma, S. Bhasin, Q. Wang, W. E. Dixon, Predictor-based control for an uncertain euler-lagrange system with input delay, Automatica 47 (2011) 2332-2342.

[5] M. Krstic, Lyapunov stability of linear predictor feedback for time-varying input delay, IEEE Trans. Autom. Control 55 (2010) 554-559.

[6] D. Bresch-Pietri, M. Krstic, Delay-adaptive predictor feedback for systems with unknown long actuator delay, IEEE Trans. Autom. Control 55 (2010) 2106-2112.

[7] Z. Lin, H. Fang, On asymptotic stability of linear systems with delayed input, IEEE Trans. Autom. Control 52 (2007) 998-1013.

[8] Z. Lin, Low Gain Feedback, Springer-Verlag, London, UK, 1988.

[9] B. Zhou, Z. Lin, G. R. Duan, Truncated predictor feedback for linear systems with long time-varying input delay, Automatica 48 (2012) 2387-2399.

[10] S. Y. Yoon, Z. Lin, Truncated predictor feedback control for exponentially unstable linear systems with time-varying input delay, Syst. \& Control Lett. 62 (2013) 837-844.

[11] S. Y. Yoon, P. Anantachaisilp, Z. Lin, An lmi approach to the control of exponentially unstable system with input time delay, in: Proc. 52nd IEEE Conf. Decis. Control, Florence, Italy, 2013, pp. 312-317.

[12] R. Herzog, P. Buhler, C. Gahler, R. Larsonneur, Unbalance compensation using generalized notch filters in the multivariable feedback of magnetic bearings, IEEE Trans. Control Syst. Technol. 4 (1996) 580-586.

[13] J. D. Setiawan, R. Mukherjee, E. H. Maslen, Adaptive compensation of sensor runout for magnetic bearings with uncertain parameters: theory and experiments, J. Dyn. Sys. Meas. Control 123 (2001) 211-218.

[14] J. D. Setiawan, R. Mukherjee, E. H. Maslen, Synchronous sensor runout and unbalance compensation in active magnetic bearings using bias current excitation, J. Dyn. Sys. Meas. Control 124 (2002) 14-24.

[15] C. Bi, D. Wu, Q. Jiang, Z. Liu, Automatic learning control for unbalance compensation in active magnetic bearings, IEEE Trans. Magn. 41 (2005) 2270-2280.

[16] I. S. Kuseyri, Robust control and unbalance compensation of rotor/active magnetic bearing systems, J. Vib. Control 18 (2012) $817-832$.

[17] J. Tang, B. Liu, J. Fang, S. S. Ge, Suppression of vibration caused by residual unbalance of rotor for magnetically suspended flywheel, J. Vib. Control 19 (2013) 1962-1979.

[18] Q. Chen, G. Liu, S. Zheng, Suppression of imbalance vibration for ambs controlled driveline system using double loop structure, J. Sound Vib. 337 (2015) 1-13.

[19] B. A. Francis, The linear multivariable regulator problem, SIAM J. Control Optim. 15 (1977) 486-505.

[20] A. Isidori, C. I. Byrnes, Output regulation of nonlinear systems, IEEE Trans. Autom. Control 35 (1990) 131-140.

[21] C. I. Byrnes, A. Isidori, Output regulation for nonlinear systems: an overview, Int. J. Robust Nonlin. Control 10 (2000) $323-337$.

[22] J. Huang, Nonlinear Output Regulation: Theory and Application, SIAM, Philadelphia, USA, 2004.

[23] M. Lu, J. Huang, A class of nonlinear internal models for global robust output regulation problem, Int. J. Robust Nonlin. Control to appear.

[24] B. Castillo-Toledo, E. Núñez-Pérez, On the regulator problem for a class of LTI systems with delays, Kibernetika 39 (4) (2003) $415-432$.

[25] D. Wang, J. Wang, P. Shi, W. Wang, Output regulation of time delay systems based on internal model principle, in: Proc. 10th IEEE Int. Conf. Control Autom. (ICCA), Hangzhou, China, 
2013, pp. 1633-1638.

[26] E. Fridman, Output regulation of nonlinear systems with delay, Syst. \& Control Lett. 50 (2003) 81-93.

[27] M. Lu, J. Huang, Robust output regulation problem for linear time-delay systems, Int. J. Control (2015) 1-12.

[28] M. Lu, J. Huang, Output regulation problem for linear timedelay systems, in: Proc. IEEE-CYBER 2014, 2014, pp. 274279 .

[29] S. Y. Yoon, Z. Lin, Robust output regulation of linear timedelay systems: A state predictor approach, J. Robust Nonlinear Controldoi:10.1002/rnc.3374.

[30] P. Anantachaisilp, L. Di, S. Y. Yoon, Z. Lin, Control of active magnetic bearing systems with input delay for applications in remotely controlled turbomachinery, in: Proc. 53rd IEEE Conf. Decis. Control, Los Angeles, CA, 2014, pp. 1197-1202.

[31] S. Y. Yoon, Z. Lin, P. Allaire, Control of Surge in Centrifugal Compressors by Active Magnetic Bearings, Springer, 2013.

[32] T. Mizuno, T. Higuchi, Design of magnetic bearing controllers based on disturbance estimation, in: Proc. 2nd Int. Symp. Magn. Bear., Tokyo, Japan, 1990, pp. 281-288.

[33] S. E. Mushi, Z. Lin, P. E. Allaire, Design, construction and modeling of a flexible rotor active magnetic bearing test rig, IEEE/ASME Trans. Mechatron. 17 (2012) 1170-1182. 